\title{
SUBMANIFOLDS, GROUP ACTIONS AND KNOTS. I
}

\author{
BY SYLVAIN E. CAPPELL AND JULIUS L. SHANESON ${ }^{1}$
}

Communicated by William Browder, March 10, 1972

This note and [4] outline new methods of classifying submanifolds of a manifold, submanifolds invariant under a group action, and submanifolds fixed under a group action. These methods solve many previously difficult problems associated with codimension two. In particular, they lead to a better understanding of the role of knot theory in the general placement problem for manifolds; this will be accomplished via the definition and computation of the local knot cobordism group of a manifold. Many of the results are most efficiently described in terms of new algebraic $K$-theoretic groups introduced in [4], [5].

$\S I$ has examples of the results on classification of embeddings of an $n$-dimensional manifold $M^{n}$ in $W^{n+2}$. This is used to solve the problem of finding a purely geometric interpretation of the periodicity of knot cobordism [7], [8], [9] and [3]. The knot cobordism groups were introduced by Milnor and Fox in the classical case [6] and computed by Kervaire and Levine in the high-dimensional case. Our methods are basically independent of theirs.

§II contains an outline of codimension 2 surgery. The obstruction groups are very large in even dimensions. Some applications to groupactions, including problems of extending free cyclic group actions and the calculation of equivariant knot cobordism are in [4], [5]. Results of this type follow from the classification theory for homology equivalent manifolds developed there.

The connection between codimension 2 problems and homology equivalent manifolds has been suggested in previous work of the authors [3] and in Santiago Lopez de Medrano [10]. A detailed exposition of this theory, which involves our systematic generalization of the nonsimply connected surgery theory and surgery groups of C.T.C. Wall is, in [5]. In [4], [5], the knot cobordism group of a manifold, or of a 2-plane bundle over a manifold, is defined and computed in terms of an algebraic $K$ theory.

AMS 1970 subject classifications. Primary 57D40, 57D65, 57C35, 57C45, 57A35, 57E30, $18 \mathrm{~F} 25,15 \mathrm{~A} \dot{6} 3$.

${ }^{1}$ The authors are Sloan Foundation fellows and were partially supported by an NSF Grant. 
Details, proofs and applications are in [5]. The results below are stated for piecewise-linear manifolds, group actions and locally-flat embedding. Analogous results are true for the differentiable and topological case,

I. Recall that two embeddings $f_{i}: X \rightarrow Y, i=0,1$, of manifolds are said to be concordant if there is an embedding $F: X \times I \rightarrow Y \times I$ with $F(i, x)=f_{i}(x), i=0,1 . G_{n}$ denotes knot cobordism group in dimension $n$, i.e., the group of concordance classes of embeddings of the $n$-sphere $S^{n}$ in $S^{n+2}$. Kervaire showed that $G_{2 k}=0$. Levine showed $G_{2 k+1}$ was an infinite direct sum of copies of $Z, Z_{2}$ and $Z_{4}$ and observed an algebraic isomorphism $G_{k} \cong G_{k+4}, k \neq 1,3$. In [3], it was proved that, for topological embeddings, $G_{k}^{\text {to }} \cong G_{k+4}^{\text {top }}, k \geqq 3$. By obtaining below two different classifications of the concordance classes of embeddings of $S^{n} \times M$ in $S^{n+2} \times M$, the problem of giving a geometric interpretation to the periodicity of knot cobordism is solved,

Let $i$ denote the usual inclusion of $S^{n}$ in $S^{n+2}$ and, for a closed manifold $M$ of dimension $k$, let $j=i \times 1_{M}: S^{n} \times M \rightarrow S^{n+2} \times M$. Two embeddings $\alpha, \beta$ of $S^{n} \times M$ in $S^{n+2} \times M$ are said to be equivalent if there are p.1. homeomorphisms $\rho_{1}: S^{n} \times M \rightarrow S^{n} \times M, \rho_{2}: S^{n+2} \times M \rightarrow S^{n+2} \times M$ with $\rho_{i}, i=1,2$, commuting up to homotopy with the projection onto $M$, with $\beta=\rho_{2} \alpha \rho_{1}, G_{n}(M)$ will denote the concordance classes of equivalent embeddings of $S^{n} \times M$ in $S^{n+2} \times M$ which are homotopic to $j$. A map $\varphi_{M}^{n}: G_{n+k} \rightarrow G_{n}(M)$ is defined by letting, for a knot $\alpha$ representing an element of $G_{n+k}, \varphi_{M}^{n}(\alpha)$ be the knot arithmetic sum of $j$ and $\alpha$.

THEOREM 1. Let $M$ be a closed simply-connected manifold of dimension $k>3$. Then $\varphi_{M}^{n}: G_{n+k} \rightarrow G_{n}(M), n>1$, is a one-to-one correspondence.

A map $P_{M}^{n}: G_{n} \rightarrow G_{n}(M)$ is defined for a knot $\alpha$ by taking its product with $M$.

THEOREM 2. Let $M$ be a closed simply-connected manifold of dimension $k=4 q$ with index \pm 1 . Then $P_{M}^{n}: G_{n} \rightarrow G_{n}(M), n>3$, is a one-to-one correspondence.

The desired geometric periodicity is now obtained by taking $M$ to be $C P^{2}$.

THEOREM 3. $\left(\varphi_{C P^{2}}^{n}\right)^{-1} P_{C P^{2}}^{n}: G_{n} \rightarrow G_{n+4}, n>3$, is an isomorphism of groups.

If $n=3,\left(\varphi_{C P^{2}}^{n}\right)^{-1} P_{C P^{2}}^{n}$ is injective with cokernel $Z_{2}$.

(For topological knots the corresponding map is an isomorphism [3].) The map $P_{M}^{n}$ is still injective if the index of $M$ is odd. Bredon [1] has a different geometric description of the periodicity $\operatorname{map} G_{n} \rightarrow G_{n+4}$. 
II. For odd-dimensional manifolds, we show that the ambient surgery obstruction in codimension two is the abstract surgery obstruction. Precisely, let $f: W \rightarrow V$ be a homotopy equivalence of closed manifolds of dimension $n$. A submanifold $M$ of $V$ determines, by making $f$ transverse to $M$, an induced surgery problem and hence, for $M$ a codimension 2 submanifold, an element $\sigma_{M}(f)$ of the Wall surgery obstruction group $L_{n-2}\left(\pi_{1} M\right)$.

THEOREM 4. If $n=2 k+1, k>2$, the map $f$ is homotopic to a map (which we continue to call $f$ ) transverse regular to $M$ with $f^{-1}(M) \rightarrow M$ a homotopy equivalence if and only if $\sigma_{M}(f)=0$. Moreover, if $\sigma_{M}(f)=0$, among the manifolds homotopy equivalent to $M$, those in one normal cobordism class, and only those, will occur as $f^{-1} M$ for some $f$ in the given homotopy class.

There is a corresponding relative form of the above result for manifolds with boundary. Recalling that $L_{2 k-1}(0)=0$, a special case is the following.

THEOREM 5. Let $f: W \rightarrow V$ be a homotopy equivalence of closed manifolds of dimension $2 k+1, k>2$. Let $M^{2 k-1}$ be a simply-connected submanifold of $V$. Then $f$ is homotopic to a map, transverse regular to $M$ (and which we continue to call $f$ ) with $f^{-1}(M) \rightarrow M$ a homotopy equivalence. Moreover, $f^{-1} M$ is uniquely determined by this.

Using the relative form of this theorem for $M=S^{2 k} \times I$ and $V=$ $S^{2 k+2} \times I$, we obtain the classical result of Kervaire on the vanishing of the even-dimensional knot cobordism groups.

The functors $\Gamma$ introduced in [4], [5] describe the obstructions to ambient codimension 2 surgery in even-dimensional manifolds. $L$ denotes the Wall surgery group functor [11]. Given $M^{2 k-2}$ a submanifold of $W^{2 k}$, there is a homomorphism

$$
\rho: L_{2 k-1}\left(\pi_{1} M\right) \rightarrow \operatorname{Ker}\left(\Gamma_{2 k}\left(Z\left[\pi_{1}(W-M)\right] \rightarrow Z\left[\pi_{1} W\right]\right) \rightarrow L_{2 k}\left(\pi_{1} W\right)\right) .
$$

THEOREM 6. Let $\left(M^{2 k-2}, \partial M\right)$ be a proper submanifold of $\left(V^{2 k}, \partial V\right)$ and let $f:(W, \partial W) \rightarrow(V, \partial V)$ be a homotopy equivalence of manifolds restricting to a homotopy equivalence $\partial W \rightarrow \partial V$. Assume, moreover, that $f^{-1}(\partial M) \rightarrow \partial M$ is a homotopy equivalence. Then $f$ is homotopic by a homotopy which is fixed on $\partial W$ to a map (which we continue to call $f$ ) with $f^{-1}(M) \rightarrow M$ a homotopy equivalence if and only if $\sigma_{M}(f)=0$ and an obstruction $\tau(f)$, defined if $\sigma_{M}(f)=0$, as an element of the cokernel of $\rho$, vanishes.

The proof of Theorem 4 uses the cobordism extension technique introduced by Browder to study embeddings in codimension greater than 2 
[2] and new methods of studying homology equivalent odd-dimensional manifolds.

\section{BIBLIOGRAPHY}

1. G. Bredon, Regular $O(n)$-manifolds, suspensions of knots, and knot periodicity (to appear).

2. W. Browder, Embedding smooth manifolds, Proc. Internat. Congress Math. (Moscow, 1966), "Mir", Moscow, 1968, pp. 712-719. MR 38 \#6611.

3. S. E. Cappell and J. L. Shaneson, Topological knots and knot cobordism topology (to appear).

4. $\frac{}{1052}$, Submanifolds, group actions and knots. II, Bull. Amer. Math. Soc. 78 (1972), 1049-1052.

5. - The codimension two placement problem and homology equivalent manifolds (to appear).

6. R. H. Fox and J. W. Milnor, Singularities of 2-spheres in 4-space and equivalence of knots, Bull. Amer. Math. Soc. 63 (1957), 406. Abstract \# 809t.

7. M. Kervaire, Les nouds de dimensions supérieures, Bull. Soc. Math. France 93 (1965), 225-271. MR 32 \#6479.

8. J. Levine, Knot cobordism groups in codimension two, Comment. Math. Helv. 44 (1968), $229-244$.

9. _ Invariants of knot cobordism, Invent. Math. 8 (1969), 98-110.

10. S. Lopez de Medrano, Invariant knots and surgery in codimension 2, Proc. Internat. Congress Math. (Nice, 1970), vol. 2, Gauthier-Villars, Paris, 1971, pp. 99-112.

11. C. T. C. Wall, Surgery of compact manifolds, London Math. Soc. Monographs, no. 1, Academic Press, New York, 1971.

Department of Mathematics, Princeton University, Princeton, New Jersey 08540

Department of Mathematics, Rutgers University, New Brunswick, New Jersey 08903 